\title{
Specific, sensitive and rapid detection of human plasmodium knowlesi infection by loop-mediated isothermal amplification (LAMP) in blood samples
}

\author{
Yee-Ling Lau", Mun-Yik Fong ${ }^{1}$, Rohela Mahmud', Phooi-Yee Chang ${ }^{1}$, Vanitha Palaeya', Fei-Wen Cheong ${ }^{1}$, \\ Lit-Chein Chin ${ }^{1}$, Claudia N Anthony ${ }^{1}$, Abdulsalam M Al-Mekhlafi ${ }^{1}$ and Yeng Chen ${ }^{2}$
}

\begin{abstract}
Background: The emergence of Plasmodium knowlesi in humans, which is in many cases misdiagnosed by microscopy as Plasmodium malariae due to the morphological similarity has contributed to the needs of detection and differentiation of malaria parasites. At present, nested PCR targeted on Plasmodium ssrRNA genes has been described as the most sensitive and specific method for Plasmodium detection. However, this method is costly and requires trained personnel for its implementation. Loop-mediated isothermal amplification (LAMP), a novel nucleic acid amplification method was developed for the clinical detection of P. knowlesi. The sensitivity and specificity of LAMP was evaluated in comparison to the results obtained via microscopic examination and nested PCR.
\end{abstract}

Methods: LAMP assay was developed based on P. knowlesi genetic material targeting the apical membrane antigen-1 (AMA-1) gene. The method uses six primers that recognize eight regions of the target DNA and it amplifies DNA within an hour under isothermal conditions $\left(65^{\circ} \mathrm{C}\right)$ in a water-bath.

Results: LAMP is highly sensitive with the detection limit as low as ten copies for AMA-1. LAMP detected malaria parasites in all confirm cases $(n=13)$ of $P$. knowlesi infection (sensitivity, 100\%) and none of the negative samples (specificity, 100\%) within an hour. LAMP demonstrated higher sensitivity compared to nested PCR by successfully detecting a sample with very low parasitaemia $(<0.01 \%)$.

Conclusion: With continuous efforts in the optimization of this assay, LAMP may provide a simple and reliable test for detecting P. knowlesi malaria parasites in areas where malaria is prevalent.

\section{Background}

Malaria still poses a major threat in most parts of the world. It is widespread in tropical and subtropical regions, including parts of America, Asia and Africa. Plasmodium knowlesi is one of the simian malaria that causes human infection. In 2004, a large focus of human P. knowlesi infection was reported in the Kapit Division of Sarawak. It was reported that 101 out of 141 (71.6\%) of human malaria cases at Kapit Hospital, which had been identified by microscopy as single Plasmodium malariae infections, were actually $P$. knowlesi and other non- $P$. malariae species by nested polymerase chain

\footnotetext{
* Correspondence: lauyeeling@um.edu.my

'Department of Parasitology, Faculty of Medicine, University of Malaya,

50603 Kuala Lumpur, Malaysia

Full list of author information is available at the end of the article
}

reaction (PCR) assays [1]. More recently, out of 111 human blood samples received (July 2006 - March 2008) by the Institute for Medical Research (IMR), 62 (55.9\%) were positive for P. knowlesi by nested PCR. Positive P. knowlesi cases were observed in most states in Peninsular Malaysia [2].

Microscopic examination has been the mainstay of malaria diagnosis. Microscopy is relatively inexpensive, rapid, and relatively sensitive procedure when used appropriately. However, interpretations of smears require considerable expertise particularly at low-level parasitaemia. This could potentially lead to false negative results or unreliable species determination. The emergence of $P$. knowlesi in humans, which is in many cases misdiagnosed by microscopy as $P$. malariae due to the similarity in morphology has contributed to the

\section{Biomed Central}

(c) 2011 Lau et al; licensee BioMed Central Ltd. This is an Open Access article distributed under the terms of the Creative Commons Attribution License (http://creativecommons.org/licenses/by/2.0), which permits unrestricted use, distribution, and reproduction in any medium, provided the original work is properly cited. 
needs of detection and differentiation of malaria parasites [3].

To date, nested PCR targeted on Plasmodium ssrRNA genes has been described as the most sensitive and specific method for Plasmodium detection. Genus- and species-specific primers have been used to amplify ssrRNA genes to detect mixed infections. However, this method is costly and requires trained personnel for its implementation.

Serology methods to detect antibodies by immunofluorescence or ELISA have been used for seroepidemiological studies of malaria. However, the difficulty of blood stage cultivation of the parasite has been hindering the use of these techniques.

Loop-mediated isothermal amplification (LAMP) which was originally developed by Notomi et al [4], is a very sensitive, easy and time saving method. The LAMP method can amplify up to $10^{9}$ copies of targeted gene in less than an hour under isothermal conditions $\left(65^{\circ} \mathrm{C}\right)$. A simple incubator, such as water bath or heating block is sufficient for the DNA amplification, which makes its use under field conditions feasible. The method uses four to six primers that recognize six to eight regions of the target DNA eliminating non-specific binding and thus ensuring the specificity of LAMP.

Of late, this method has been proven to be a powerful diagnostic tool in the detection of many parasitic infections in both human and animal models. In this study, a LAMP assay was developed for the detection of P. knowlesi genetic material targeting the apical membrane antigen-1 (AMA-1) gene. AMA-1 found in all Plasmodium parasites during the late schizont stage. This protein was reported to play a prominent role in the invasion of the host cell [5].

\section{Methods}

\section{Clinical samples}

Fifty-four malaria positive blood samples were collected from University Malaya Medical Centre (UMMC), Malaysia. Samples were further diagnosed by microscopy and nested PCR assay. Twenty blood samples were also collected from healthy donor. Ethical approval for this study was obtained from the Medical Ethics Committee of University Malaya Medical Centre.

\section{Microscopy}

Thick and thin blood films were prepared and examined by skilled personnel with extensive experience in the identification of malaria parasites. Parasitaemia was assessed either per 1,000 erythrocytes in the thin film at low parasitaemia or per 200 white blood cells (WBC) in the thick film. In case of a putative negative film, it was considered negative if no parasites were seen after 500 leukocytes were counted.

\section{Rapid diagnostic kit}

BinaxNOW Malaria Kit (Inverness Medical International, United Kingdom) was performed according to manufacturer's instructions and adapted using a drop of EDTA anticoagulated whole blood from microscopy confirmed cases. Test results were analysed after 15 minutes as recommended by the manufacturer's manual.

\section{DNA Extraction}

Template DNA for LAMP and nested PCR assay were prepared using DNeasy Blood \& Tissue Kit (QIAGEN, Valencia, CA). EDTA anticoagulated whole blood (200 $\mu \mathrm{l})$ was extracted according to the provided protocol to give $100 \mu \mathrm{l}$ of purified template DNA. Purified DNA was stored at $-20^{\circ} \mathrm{C}$.

\section{Nested PCR assay}

The species of the malaria samples were determined by nested PCR assay. This assay targets the Plasmodium small subunit ribosomal RNA (ssrRNA) gene. The primers used for the nested PCR assay were identical to those previously published [1]. The reaction mixture for the first PCR step had $2 \mu \mathrm{l}$ of DNA template, $250 \mathrm{nmol} /$ $\mathrm{L}$ of each primer (rPLU 1 and $r P L U$ 5, PCR buffer (50 $\mathrm{mmol} / \mathrm{L} \mathrm{KCl}, 10 \mathrm{mmol} / \mathrm{L}$ Tris $-\mathrm{HCl}), 200 \mathrm{mmol} / \mathrm{L}$ of each deoxynucleoside triphosphate, 1.25 units of Taq DNA polymerase (iDNA, Singapore), and water to a final volume of $25 \mu \mathrm{l}$. Primary amplification conditions were $94^{\circ} \mathrm{C}$ for 4 minutes; 35 cycles at $94^{\circ} \mathrm{C}$ for $30 \mathrm{sec}-$ onds, annealing at $55^{\circ} \mathrm{C}$ for 1 minute, extension at $72^{\circ} \mathrm{C}$ for 1 minutes; and final extension at $72^{\circ} \mathrm{C}$ for 4 minutes. Two microliters of the first amplification product was used as DNA template for each of the $20 \mathrm{ml}$ secondary amplifications. The conditions and concentrations of the secondary amplification were identical to those of the primary except for the annealing temperature of $58^{\circ} \mathrm{C}$ and the amount of Taq Polymerase being 0.5 units. The PCR products of the secondary amplification were analysed by gel electrophoresis and stained with ethidium bromide.

\section{LAMP assay}

The FIP, BIP, F3, and B3 primers were designed using the Primer-Explorer V3 software [6] based on the apical membrane antigen-1 (AMA-1) gene sequence of $P$. knowlesi (GenBank accession number XM_002259303) (Table 1). Loop primers (Loop-F and Loop-B) were designed manually. The $25 \mu \mathrm{L}$ reaction mixture consisted of $1.6 \mu \mathrm{M}$ FIP and BIP, $0.8 \mu \mathrm{M}$ Loop-F and LoopB, $0.2 \mu \mathrm{M}$ F3 and B3, $20 \mathrm{mM}$ Tris- $\mathrm{HCl}, 10 \mathrm{mM} \mathrm{KCl}, 8$ $\mathrm{mM}$ MgSO4, 10 mM NH4SO4, 0.1\% Tween 20, $0.8 \mathrm{M}$ betaine, $1.4 \mathrm{mM}$ deoxynucleotide triphosphates, $1 \mu \mathrm{L}$ Bst DNA polymerase and $2 \mu \mathrm{L}$ template DNA. The reaction mixture was incubated in a water bath at $65^{\circ} \mathrm{C}$ 


\section{Table 1 Primers used in this study}

\begin{tabular}{ll}
\hline Primer & Sequence $\left(\mathbf{5}^{\prime} \rightarrow \mathbf{3}^{\prime}\right)$ \\
\hline FIP & TTACAAACGTAAAAGTTGCAGGTACGATAAGGAGAGTATCAA \\
ATGTCCA & CTGTGTAGAGAAGAGAGCAGAAATCCGGATTTCATAATCCTCC \\
F3 & GCCAAGGATATTATCTCCAC \\
B3 & CTTCTTCTTATGTTTGCCGT \\
Loop-F & GGAAATGTGTTCAGGCTCAC \\
Loop-B & CATAAAGGAAGAATTAA \\
\hline
\end{tabular}

for 45-60 minutes. To prevent cross-contamination, different sets of pipettes and different work areas were designated for DNA template preparation, PCR mixture preparation and DNA amplification.

\section{Endpoint assessment}

Turbidity of the reaction mixture was observed with the naked eye. Turbidity is based on the precipitation of magnesium pyrophosphate as a by-product of the reaction. Besides the assessment of turbidity by the naked eye, amplicons of the LAMP were also detected by adding $1.0 \mu \mathrm{L}$ of $1 / 10$-diluted original $\mathrm{SYBR}^{\circledR}$ Safe DNA gel stain (Invitrogen, Carlsbad, CA) to the tubes and observed under UV. Solutions with amplicons showed stronger luminescence under UV compared to the solutions without amplicons. For further confirmation, some of the amplified LAMP products were analysed by gel electrophoresis. All reactions were analysed by $2.0 \%$ (wt/ vol) agarose gel by electrophoresis in Tris-AcetateEDTA (TAE) buffer stained with SYBR ${ }^{\circledR}$ Safe DNA gel stain and positive results were identified by the appearance of typical ladder bands of various sizes.

\section{Positive control plasmid DNA}

For sensitivity assessment, plasmids containing the target region of the AMA gene were constructed for $P$. knowlesi for use in the LAMP reaction. The target DNA sequence was amplified with two LAMP primers (primers F3 and B3) by PCR and was then cloned into the pGEMT cloning vector (Invitrogen, CA). Plasmid DNA purification was performed with a QIAprep Miniprep kit (QIAGEN, Hilden, Germany). The resulting sequences were aligned using the AMA sequences for $P$. knowlesi in GenBank to confirm that the target sequences were correct.

\section{Analytical sensitivity of LAMP primers}

Positive control plasmid DNAs were used to determine the minimum copy number (lower detection limit) of the target gene sequence detectable by LAMP. The standard curve for LAMP was constructed using 10-fold serial dilutions of plasmid DNA $\left(10^{6}\right.$ copies to 1 copy) to sterile water. The copy number was plotted against the threshold time. The resulting plot was analysed by linear regression and the statistical significance of the $r 2$ values was analysed by analysis of variance (ANOVA). Probabilities of less than 0.05 were considered statistically significant.

\section{Analytical specificity of LAMP primers}

Specificity of the LAMP primers were tested using genomic DNAs (gDNAs) of various Plasmodium species $(P$. knowlesi, Plasmodium falciparum, Plasmodium simium, Plasmodium cynomolgi, Plasmodium fragile, Plasmodium brasilianum, Plasmodium vivax) by gel electrophoresis. The gDNAs were obtained from American Type Culture Collection (ATCC).

\section{Clinical sensitivity and specificity}

The clinical sensitivity and specificity of LAMP assay was calculated using 74 whole-blood samples and microscopy as the reference standard method. Sensitivity was calculated as (number of true positives)/(number of true positives + number of false negatives), and specificity was calculated as (number of true negatives)/(number of true negatives+ number of false positives).

\section{Results}

A total of 74 samples were included in this study to determine the sensitivity and specificity of LAMP method in detecting P. knowlesi.

\section{Specificity of LAMP primers}

Various plasmodium genomic DNAs comprising of 39 non-knowlesi malaria blood samples ( $P$. vivax, $\mathrm{n}=28 ; P$. falciparum, $\mathrm{n}=10 ; P$. malariae, $\mathrm{n}=1$, as determined by BinaxNOW Malaria Kit and nested PCR) and 20 blood samples from healthy donors were used as template in this LAMP experiment to investigate the specificity of LAMP primers. All P. knowlesi positive samples produced a typical ladder of multiple bands on the agarose gel while other plasmodium and healthy donor samples did not produce such bands (Figure 1).

\section{Sensitivity of LAMP detection}

LAMP is highly sensitive with the detection limit as low as ten copies for AMA-1 plasmid compared to nested PCR (100 copies). Clinical sensitivity of LAMP was compared with the results from conventional nested PCR and microscopy. By microscopy, a total of 13 P. knowlesi suspected samples (12 with either $P$. malarie or $P$. knowlesi and 1 with either P. falciparum or P. knowlesi) were detected positive for $P$. knowlesi under LAMP. However, nested PCR with species specific primers only detected 12 samples as positive for P. knowlesi while another sample showed no relation to other Plasmodium species (Table 2). 


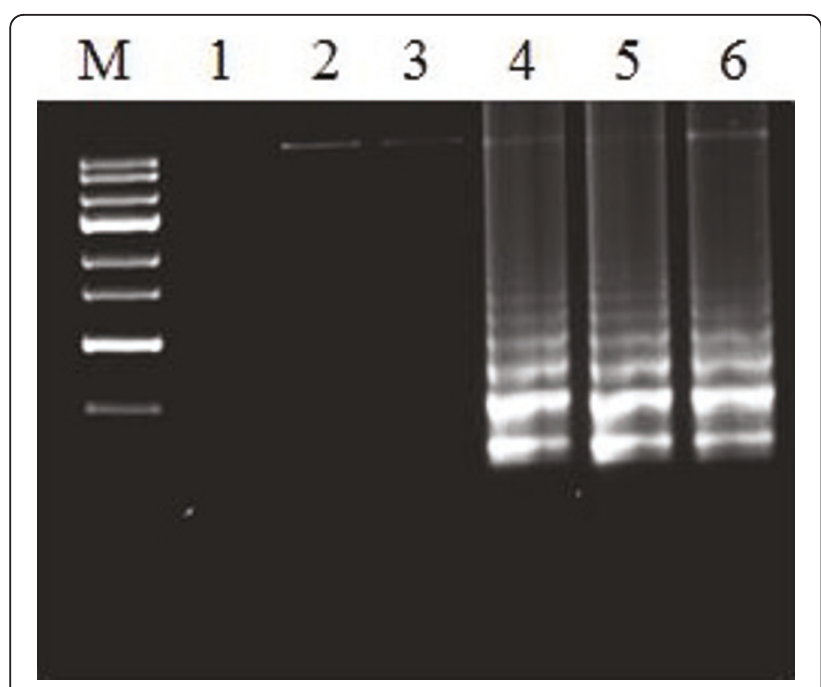

Figure 1 Agarose gel electrophoresis of LAMP products. Electrophoresis of loop-mediated isothermal amplification (LAMP) products amplified from genomic DNA and blood samples. Lane 13, negative controls of genomic DNA of $P$. falciparum, genomic DNA $P$. vivax and blood sample of healthy donor, respectively; lane 4, genomic DNA of P. knowlesi; lane 5-6, P. knowlesi positive blood samples and lane M, 100-bp DNA ladder.

\section{Optimization of LAMP assay}

Temperature of $65^{\circ} \mathrm{C}$ was found to be optimal in producing the best ladder pattern for positive P. knowlesi samples. Similar results were obtained when either a water bath or a heating block was used.

The LAMP assay was easy to conduct, although prevention of contamination proved necessary. Precautions such as changing of gloves between every PCR assay and different work areas for different parts of the experiment were taken into account.

\section{Discussion}

Loop-mediated isothermal amplification (LAMP), the novel method developed by Notomi et al [4], is able to amplify DNA with great efficacy as its detection limit for DNA in the reaction mixture is as low as six copies, within an hour under isothermal conditions $\left(65^{\circ} \mathrm{C}\right)$. LAMP is also an easy, convenient and cost-saving method, which only requires simple laboratory apparatus such as water bath or heating blocks to perform the test. Therefore, it has been widely applied as a diagnostic tool for several viral, bacterial, and parasitic diseases [7-9]. Han et al had reported a species-specific LAMP method for the diagnosis of four well-known human malaria Plasmodium species [10]. This study is the first report whereby LAMP assay is used for the diagnosis of the fifth human malaria $P$. knowlesi infection in human blood samples. In this study, P. knowlesi DNA was successfully amplified within an hour at $65^{\circ} \mathrm{C}$ by using LAMP primers that target the AMA gene of P. knowlesi.

Previous studies have indicated that LAMP assay has a higher sensitivity compared to nested PCR in detection of parasites, such as Trypanosoma spp. [11], Babesia spp. [12], and T. gondii [9]. The capability of LAMP assay to detect a single microfilaria in samples, as demonstrated by Aonuma et al, clearly shows that the method is highly sensitive [13]. In order to evaluate the sensitivity of LAMP assay for the detection of P. knowlesi in human samples, the LAMP assay was compared with conventional nested PCR, which targeted on $P$. knowlesi SSU gene and microscopic examination. Under microscopy, the early trophozoites of $P$. knowlesi morphologically resembled those of $P$. falciparum, while the late and mature trophozoites, schizonts and gametocytes of $P$. knowlesi in human infections were generally

Table 2 Results of LAMP compared to microscopy and nested PCR for detecting $\boldsymbol{P}$. knowlesi

\begin{tabular}{|c|c|c|c|}
\hline \multirow[t]{2}{*}{ Microscopy/nested PCR result } & \multicolumn{2}{|c|}{ LAMP result } & \multirow[t]{2}{*}{ Microscopy/nested PCR total } \\
\hline & P. knowlesi & Negative & \\
\hline \multicolumn{4}{|l|}{ Microscopy result } \\
\hline P. knowlesi or P. malariae & 12 & $1^{\mathrm{a}}$ & 13 \\
\hline P. knowlesi or P. falciparum & $1^{c}$ & $1^{\mathrm{b}}$ & 2 \\
\hline Non-knowlesi & 0 & 39 & 39 \\
\hline Negative & 0 & 20 & 20 \\
\hline \multicolumn{4}{|l|}{ Nested $P C R$ result } \\
\hline P. knowlesi & 12 & 0 & 12 \\
\hline Non-knowlesi & 0 & 41 & 41 \\
\hline Negative & $1^{c}$ & 20 & 21 \\
\hline LAMP total & 13 & 61 & 74 \\
\hline
\end{tabular}

\footnotetext{
a Sample positive for P. malariae by nested PCR

b Sample positive for $P$. falciparum by nested PCR

c Sample positive for either $P$. knowlesi or $P$. falciparum by microscopy, with parasitaemia less than $0.01 \%$
} 
indistinguishable from those of $P$. malariae. Hence, the lack of distinguishable morphological features of $P$. knowlesi makes it extremely difficult to identify P. knowlesi infections by microscopy alone and laboratory misdiagnosis of $P$. knowlesi as P. malariae is inevitable [3].

In this study, 13 patient samples which were suspected to be either P. knowlesi/P. malariae or P. knowlesi/P. falciparum infections and were unable to be determined solely by microscopic examination, showed positive for $P$. knowlesi via LAMP assay, while P. knowlesi nested PCR was unable to detect one of these cases (12 positive as $P$. knowlesi infections among the 13 samples). The parasitaemia level of that particular case was found to be very low $(<0.01 \%)$. On the other hand, there were two cases whereby microscopic examination showed it to be $P$. knowlesi/P. malariae or P. knowlesi/P. falciparum infection but both LAMP and nested PCR showed negative for P. knowlesi. This might be due to misidentification by using microscopic examination as it requires training, skills and experience. These results showed that LAMP method which employs four primers and targets six distinct sequences on the $P$. knowlesi DNA could be used as a molecular confirmatory test of $P$. knowlesi infection instead of microscopic examination which is unable to differentiate the species among P. knowlesi, P. malariae and $P$. falciparum. Furthermore, it is more sensitive than nested PCR and the detection limit of LAMP assays is also lower relative to nested PCR.

Besides sensitivity, the high specificity of LAMP assay was demonstrated by screening genomic DNAs other Plasmodium species (P. falciparum, P. simium, P. cynomolgi, Plasmodium fragile, P. brasilianum, Plasmodium inui and Plasmodium simiovale), and extracted DNA from blood samples of other Plasmodium malaria and healthy donors. Results showed that LAMP assay which was specifically designed for P. knowlesi did not amplify DNAs of other Plasmodium spp. and no cross-reactivity occurred with all the negative controls. The result indicates that by using LAMP assays, $P$. knowlesi can be efficiently distinguished from other malaria parasites.

The simplicity and high efficiency of LAMP to amplify DNA under isothermal conditions within an hour suggests that LAMP could be a potential alternative for detection of P. knowlesi in samples compared to nested PCR, which requires an expensive PCR machine and is time-consuming. Another advantage of using LAMP assay is due to its turbidity-based detection of the positive reaction. The positive and negative amplifications could be distinguished by observing the turbidity of reactions with the naked eye, without using gel electrophoresis and therefore the result can be analysed within a few minutes [14]. Another way to analyse the LAMP result is by adding a DNA intercalating dye, SYBR green I to the end-products and visualizing it under UV light.
The turbidity can be better observed under UV light. Besides that, the amplicon sequence was confirmed by sequence analysis to eliminate false positive and contaminated reactions.

There are however, limitations to this method. For visualization of results under UV light, the product tubes need to be opened for the dye to be added and this might lead to cross-contamination. This limitation can be overcome by practicing sterile pipetting techniques or using LAMP master mix, which contains preadded fluorescence dye. Another drawback is the size of target DNA. The efficiency of LAMP is affected by the size of target DNA as the strand displacement DNA synthesis step limits the rate or amplification. The size of target DNA has to be lesser than 300 base pair in order to obtain satisfying results [4]. Hence, target DNA, which is larger than $500 \mathrm{bp}$ is not suitable to be used as it will lead to poor amplification.

\section{Conclusion}

Based on the results obtained, LAMP assays could be a potential alternative for molecular diagnosis and routine screening of P. knowlesi infection especially in malaria endemic countries, including Malaysia. It could also be useful in monitoring malaria control and eradication programmes.

\section{Acknowledgements}

The study was supported by University of Malaya, High Impact Research Fund (Grant No. J-20011-73592) and Ministry of Science, Technology \& Innovation (MOSTI), E-Science Fund (Grant No. 02-01-03-SF0602). Special thanks to PARASEAD, UM for providing part of the blood samples for analysis.

\section{Author details}

'Department of Parasitology, Faculty of Medicine, University of Malaya, 50603 Kuala Lumpur, Malaysia. ${ }^{2}$ Institute for Research in Molecular Medicine (INFORMM), Universiti Sains Malaysia, 11800 Penang, Malaysia.

\section{Authors' contributions}

YLL, RM, PYC, VP, FWC, LCC and YC carried out laboratory work and analysed the data. LCC and AMA collected the blood samples. MYF and CNA participated in the data analyses and helped to draft the manuscript. All authors read and approved the final manuscript.

\section{Competing interests}

The authors declare that they have no competing interests.

Received: 6 May 2011 Accepted: 20 July 2011 Published: 20 July 2011

\section{References}

1. Singh B, Kim Sung L, Matusop A, Radhakrishnan A, Shamsul SS, Cox-Singh J, Thomas A, Conway DJ: A large focus of naturally acquired Plasmodium knowlesi infections in human beings. Lancet 2004, 363:1017-1024.

2. Vythilingam I, Noorazian YM, Huat TC, Jiram Al, Yusri YM, Azahari AH, Norparina I, Noorrain A, Lokmanhakim S: Plasmodium knowlesi in humans, macaques and mosquitoes in peninsular Malaysia. Parasit Vectors 2008, $1: 26$.

3. Lee KS, Cox-Singh J, Singh B: Morphological features and differential counts of Plasmodium knowlesi parasites in naturally acquired human infections. Malar J 2009, 8:73. 
4. Notomi T, Okayama H, Masubuchi H, Yonekawa T, Watanabe K, Amino N, Hase T: Loop-mediated isothermal amplification of DNA. Nucleic Acids Res 2000, 28:E63.

5. Triglia T, Healer J, Caruana SR, Hodder AN, Anders RF, Crabb BS, Cowman AF: Apical membrane antigen 1 plays a central role in erythrocyte invasion by Plasmodium species. Mol Microbiol 2000 38:706-718.

6. LAMP primer designing software PrimerExplorer. [http://primerexplorer.jp/ e/].

7. Parida M, Posadas G, Inoue S, Hasebe F, Morita K: Real-time reverse transcription loop-mediated isothermal amplification for rapid detection of West Nile virus. J Clin Microbiol 2004, 42:257-263.

8. Imai M, Ninomiya A, Minekawa H, Notomi T, Ishizaki T, Tashiro M, Odagiri T: Development of H5-RT-LAMP (loop-mediated isothermal amplification) system for rapid diagnosis of $\mathrm{H} 5$ avian influenza virus infection. Vaccine 2006, 24:6679-6682.

9. Lau YL, Meganathan P, Sonaimuthu P, Thiruvengadam G, Nissapatorn V, Chen Y: Specific, sensitive, and rapid diagnosis of active toxoplasmosis by a loop-mediated isothermal amplification method using blood samples from patients. J Clin Microbiol 2010, 48:3698-3702

10. Han ET, Watanabe R, Sattabongkot J, Khuntirat B, Sirichaisinthop J, Iriko H, Jin L, Takeo S, Tsuboi T: Detection of four Plasmodium species by genusand species-specific loop-mediated isothermal amplification for clinical diagnosis. J Clin Microbiol 2007, 45:2521-2528.

11. Kuboki N, Inoue N, Sakurai T, Di Cello F, Grab DJ, Suzuki H, Sugimoto C, Igarashi I: Loop-mediated isothermal amplification for detection of African trypanosomes. J Clin Microbiol 2003, 41:5517-5524.

12. Iseki H, Alhassan A, Ohta N, Thekisoe OM, Yokoyama N, Inoue N, Nambota A, Yasuda J, Igarashi I: Development of a multiplex loopmediated isothermal amplification (mLAMP) method for the simultaneous detection of bovine Babesia parasites. J Microbiol Methods 2007, 71:281-287.

13. Aonuma H, Suzuki M, Iseki H, Perera N, Nelson B, Igarashi I, Yagi T, Kanuka $\mathrm{H}$, Fukumoto S: Rapid identification of Plasmodium-carrying mosquitoes using loop-mediated isothermal amplification. Biochem Biophys Res Commun 2008, 376:671-676.

14. Mori Y, Nagamine K, Tomita N, Notomi T: Detection of loop-mediated isothermal amplification reaction by turbidity derived from magnesium pyrophosphate formation. Biochem Biophys Res Commun 2001, 289:150-154

doi:10.1186/1475-2875-10-197

Cite this article as: Lau et al.: Specific, sensitive and rapid detection of human plasmodium knowlesi infection by loop-mediated isothermal amplification (LAMP) in blood samples. Malaria Journal 2011 10:197.

\section{Submit your next manuscript to BioMed Central and take full advantage of:}

- Convenient online submission

- Thorough peer review

- No space constraints or color figure charges

- Immediate publication on acceptance

- Inclusion in PubMed, CAS, Scopus and Google Scholar

- Research which is freely available for redistribution

Submit your manuscript at www.biomedcentral.com/submit 